\title{
Molécules polaires ultrafroides dans le régime quantique
}

Deborah S. Jin et Jun Ye

Membres du NIST ${ }^{(1)}$ et du JILA (2), et professeurs de physique à I'Université du Colorado à Boulder

(1) NIST : National Institute of Standards and Technology

(2) JILA : Joint Institute University of Colorado/NIST, 440 UCB, Boulder, C0 80309, USA

Reproduit, avec permission, à partir de l'article original “Polar molécules in the quantum régime”, par D.S. Jin et J. Ye, Physics Today 64 (mai 2011), pp. 27-31.

Traduction en français par A. Chotia et 0 . Dulieu.

Copyright 2011, American Institute of Physics.

L'obtention en laboratoire

de gaz moléculaires à des

températures proches du

zéro absolu permet d'étudier

des réactions chimiques

comme elles ne l'ont jamais

été auparavant.

Dans cet article, nous présen-

tons un aperçu de certaines

des techniques impliquées

dans la création, le contrôle

et la compréhension des

molécules ultrafroides ;

nous décrivons aussi la première

série d'expériences qui

démontrent l'existence de

collisions moléculaires et de

réactions chimiques ultrafroides,

dans un régime où elles doivent

être décrites en termes de

fonctions d'ondes quantiques.

Les termes en italiques gras suivis d'un astérisque sont définis dans le glossaire, p. 18.
Les recherches sur les molécules à ultrabasse température (c'est-à-dire dont l'énergie cinétique est équivalente à une température très inférieure au millikelvin) représentent une nouvelle interface de la physique atomique et moléculaire et de l'optique avec l'information quantique, la chimie, la physique de la matière condensée et l'astrophysique [1]. Les molécules étant les éléments constitutifs d'un grand nombre de matériaux, le contrôle des interactions moléculaires est une quête scientifique de première importance pour des générations de physiciens.

Au cours des trente dernières années, de nombreuses équipes de recherche sont parvenues à créer des gaz quantiques dégénérés où les atomes ultrafroids, pratiquement immobiles, révèlent des comportements collectifs remarquables comme la condensation de Bose-Einstein. Atteindre le même objectif avec des molécules ultrafroides ouvre de nouveaux horizons, qui impliquent la prise en compte de la complexité intrinsèque des molécules résultant de leurs nombreux degrés de liberté internes (vibrationnels, rotationnels, structure fine et hyperfine...).

\section{Les molécules froides: pour quoi faire?}

Dans un gaz moléculaire ultrafroid à une température voisine de 0,1 microkelvin, l'énergie interne des états de vibration peut être jusqu'à dix ordres de grandeur plus élevée que l'énergie cinétique typique du mouvement de translation (fig. 1). Le contrôle de la population des états quantiques internes et du flux d'énergie de ces états vers le mouvement de translation revêt donc une importance primordiale lors de la préparation d'un gaz de molécules ultrafroides, si l'on veut exploiter la richesse de leur structure interne.

Les expériences avec des molécules représentent une extension naturelle de celles effectuées avec des systèmes atomiques. En particulier, des mesures de spectroscopie sur des molécules ultrafroides peuvent atteindre un niveau de résolution et de précision tel qu'elles constituent des tests cruciaux de certaines lois fondamentales de la physique, comme l'invariance des constantes fondamentales, les brisures de symétrie, la violation de la parité, et des possibles extensions du modèle standard $[1,2,3]$.

Les molécules dites polaires ${ }^{\star}$ sont facilement manipulables par des champs électriques externes, de sorte que leurs moments dipolaires peuvent être orientés dans le référentiel du laboratoire. Leur interaction présente alors une anisotropie significative pour les distances typiques entre particules dans un gaz ultrafroid ; ceci offre de nouvelles possibilités d'études d'effets quantiques collectifs [4, 5]. De tels gaz quantiques moléculaires mettent en jeu des effets à $\mathrm{N}$ corps (et non plus binaires) induits par l'interaction dipôle-dipôle, qui conduisent entre autres (i) à la création de superfluides composés de molécules fermioniques, 
La tour JILA à I'Université du Colorado à Boulder, au pied des montagnes Rocheuses. JILA, géré conjointement par l'Université du Colorado et le NIST, est l'un des instituts de recherche en sciences physiques les plus importants des États-Unis. Ses principaux domaines de recherche sont la physique atomique et moléculaire, l'optique de précision et les lasers, et l'astrophysique. Elle compte dans ses membres trois prix Nobel (Eric Cornell, John L. Hall et Carl Wieman).

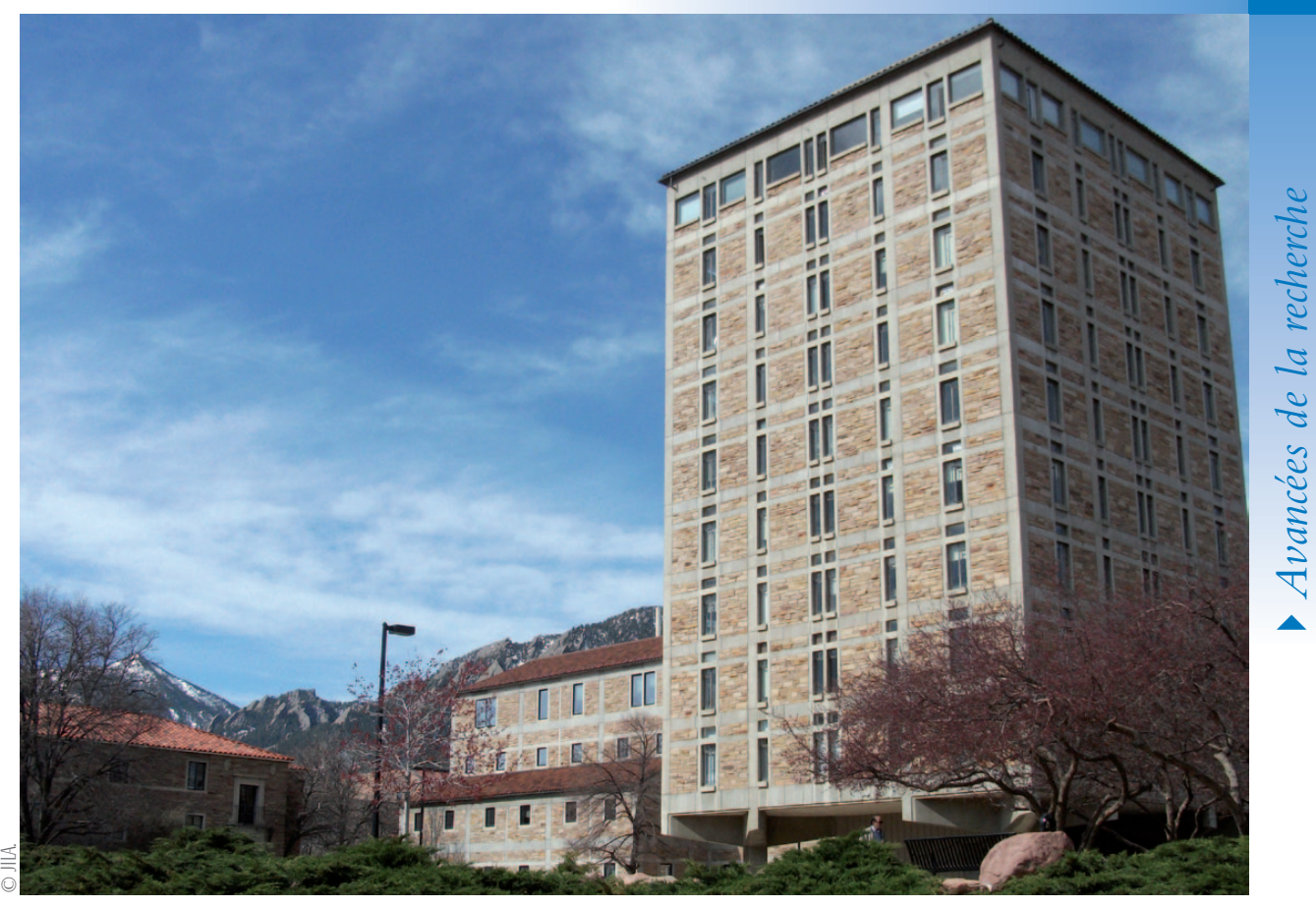

\section{Refroidir des molécules, ou les créer déjà froides ?}

La qualité de la préparation de gaz moléculaires ultrafroids est essentielle pour explorer cette thématique scientifique au travers des exemples ci-dessus. Une spectroscopie de très haute précision requiert le contrôle des états quantiques de translation des molécules, tandis que l'obtention de températures les plus basses possibles conduit à des temps de cohérence longs. L'étude des nouveaux systèmes quantiques nécessite que le gaz moléculaire ultrafroid soit dense* (typiquement $10^{12}$ à $10^{14}$ molécules $/ \mathrm{cm}^{3}$ ), stable et facilement polarisable. La caractérisation des réactions chimiques ultrafroides suppose que l'on puisse créer initialement des molécules dans un état quantique individuel parfaitement déterminé.
Plusieurs méthodes de refroidissement de molécules polaires ont récemment été proposées $[2,3]$ : (i) par contact thermique avec un gaz atomique refroidi par cryogénie, tel que l'hélium ; (ii) par ralentissement d'un jet moléculaire jusqu'à l'arrêt dans le référentiel du laboratoire, en appliquant des champs électriques, magnétiques ou optiques spatialement inhomogènes et synchrones agissant sur le moment dipolaire intrinsèque de la molécule [7] ; (iii) par laser, méthode qui reste toutefois difficile en raison de la dispersion de la population vers les nombreux niveaux d'énergie moléculaires au cours du processus de pompage optique, rendant la plupart des molécules insensibles au laser de refroidissement. Jusqu'à présent, ces techniques de refroidissement direct ont produit des gaz moléculaires avec une densité dans l'espace des phases * inférieure à $10^{-12}$, là
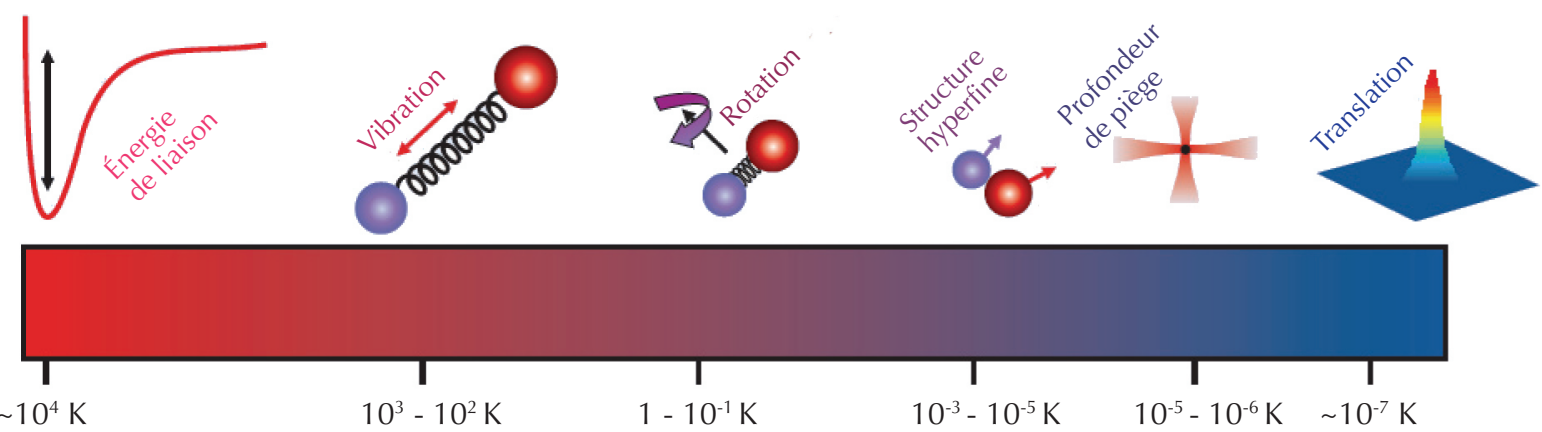

1. Degrés de liberté moléculaire et échelles d'énergie correspondantes, qui couvrent plus de dix ordres de grandeur dans un gaz quantique ultrafroid. 


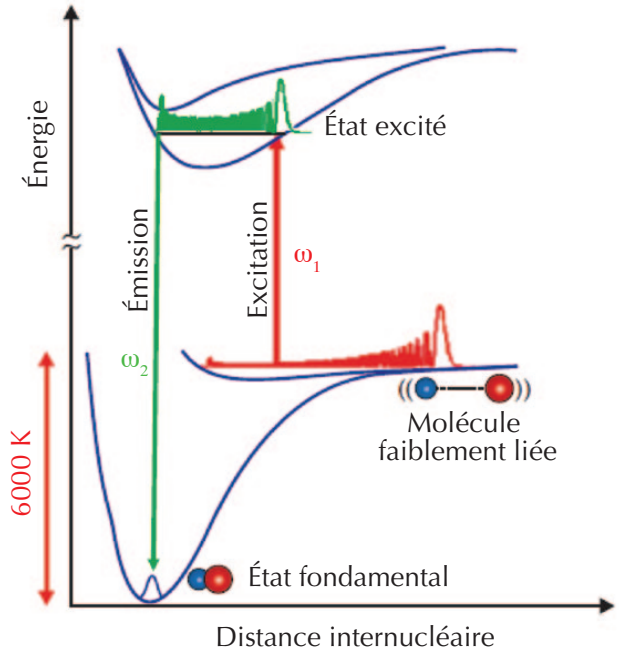

2. Schéma de principe de formation de molécules polaires froides KRb dans leur état ro-vibrationnel fondamental, par transfert cohérent de la population depuis un niveau faiblement lié créé par association d'atomes froids au moyen d'un champ magnétique. Les courbes oscillantes sont les fonctions d'ondes du mouvement radial relatif des atomes dans les potentiels moléculaires (courbes bleues). (Adapté de la réf. [2]).

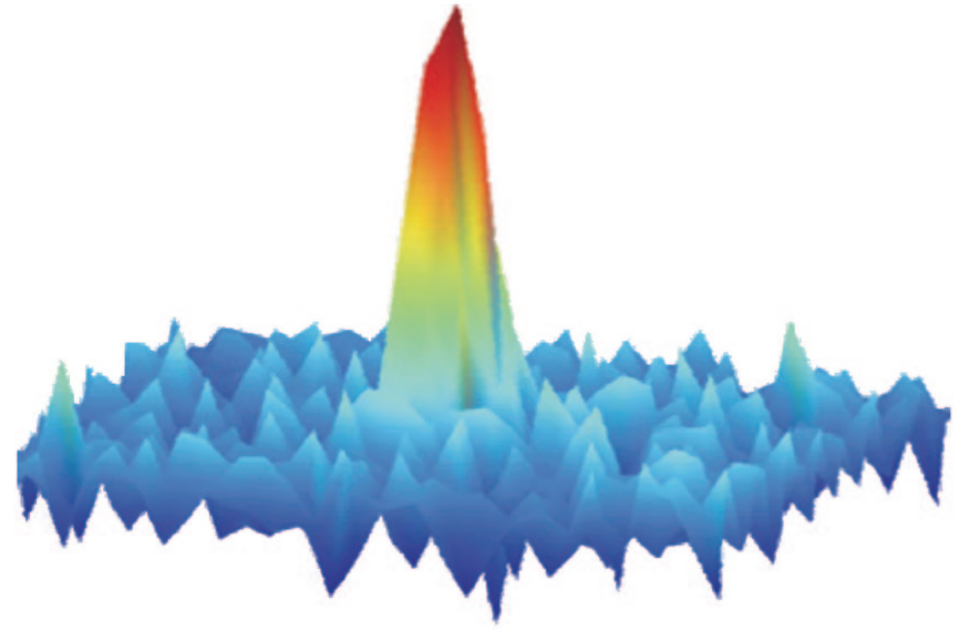

3. Image de la densité spatiale d'un gaz ultrafroid de molécules de KRb, obtenue par absorption lumineuse à $660 \mathrm{~nm}$ en résonance avec une transition moléculaire.

\section{$\gg>$}

où une valeur de l'ordre de 1 est nécessaire pour obtenir un gaz quantique dégénéré. Les gaz obtenus ont néanmoins permis de réaliser des études de spectroscopie à haute résolution, et d'observer des collisions à faible énergie en régime semi-classique.

Une alternative au refroidissement direct de molécules consiste à associer par laser une paire d'atomes ultrafroids (par exemple des alcalins, que l'on sait refroidir dans ce domaine), formant ainsi une molécule ultrafroide. Ce processus de "photoassociation " repose sur une transition optique depuis un état quantique constitué de deux atomes en collision vers un niveau ro-vibrationnel * d'un état moléculaire électroniquement excité, qui se désexcite ensuite par émission spontanée vers les niveaux ro-vibrationnels de l'état électronique fondamental de la molécule. Cependant, de par son caractère aléatoire, cette dernière étape laisse les molécules dans une large distribution de niveaux, de sorte que la densité typique dans l'espace des phases est de nouveau inférieure à $10^{-12}$.

L'efficacité de la formation de molécules dans un état quantique unique peut être augmentée en rendant le processus entièrement cohérent (fig. 2), conservant ainsi la densité dans l'espace des phases du gaz atomique ultrafroid. De récentes avancées expérimentales dans le domaine des gaz atomiques ultrafroids et des lasers de précision ont permis de mettre en œuvre ce processus cohérent, en partant par exemple d'un gaz quantique ultrafroid contenant deux espèces atomiques, du potassium (isotope fermionique ${ }^{40} \mathrm{~K}$ ) et du rubidium (isotope bosonique ${ }^{87} \mathrm{Rb}$ ). La première étape consiste à produire efficacement des molécules très faiblement liées (qui ne peuvent exister que dans le régime ultrafroid) dans un état quantique interne unique, non pas avec un laser, mais en tirant parti d'une résonance de diffusion dite de Fano-Feshbach*. Cette résonance est atteinte expérimentalement à l'aide d'un champ magnétique, qui égalise l'énergie cinétique relative de la paire d'atomes initiale et celle de la molécule faiblement liée. Puis une paire de lasers verrouillés en phase sur un peigne de fréquence optique, est utilisée pour transférer de manière cohérente ces molécules vers leur état fondamental par l'intermédiaire d'un état électronique excité (fig. 2).

Cette technique a permis d'obtenir un gaz de molécules fermioniques ${ }^{40} \mathrm{~K}^{87} \mathrm{Rb}$ d'une température de quelques centaines de nanokelvins, et de densité spatiale de $10^{12}$ molécules par centimètre cube, correspondant à une densité dans l'espace des phases d'environ 0,1. Ce gaz de molécules ultrafroides peut être imagé en utilisant un laser en résonance avec une transition moléculaire (fig. 3).

\section{Collisions et chimie froides}

Cette densité élevée dans l'espace des phases $(\sim 0,1)$ nous rapproche de la création d'un gaz quantique de molécules polaires, présentant de fortes interactions anisotropes. La température du gaz de KRb obtenue est seulement 1,5 fois la température de Fermi, en dessous de laquelle débute le régime quantique.

Une condition préalable pour atteindre le régime quantique repose sur la compréhension des collisions moléculaires ultrafroides. D'une part, le taux de collisions élastiques détermine le temps nécessaire pour que le gaz retrouve un équilibre thermique après une perturbation. D'autre part, le taux de collisions inélastiques fixe la durée de vie du gaz piégé. Le premier doit dominer le second, de sorte que les collisions élastiques entraînent l'évaporation du gaz (comme dans une tasse de café chaud), conduisant à une température plus basse et à une densité plus forte. En outre, ces collisions peuvent être réactives, ouvrant la voie à cette nouvelle chimie ultrafroide évoquée plus haut, dominée par les interactions à très grande distance, par les effets de statistique quantique au travers du spin nucléaire et de la structure hyperfine des molécules (habituellement ignorée en chimie), et où les taux de réaction sont régis par des « lois de seuil » universelles. 


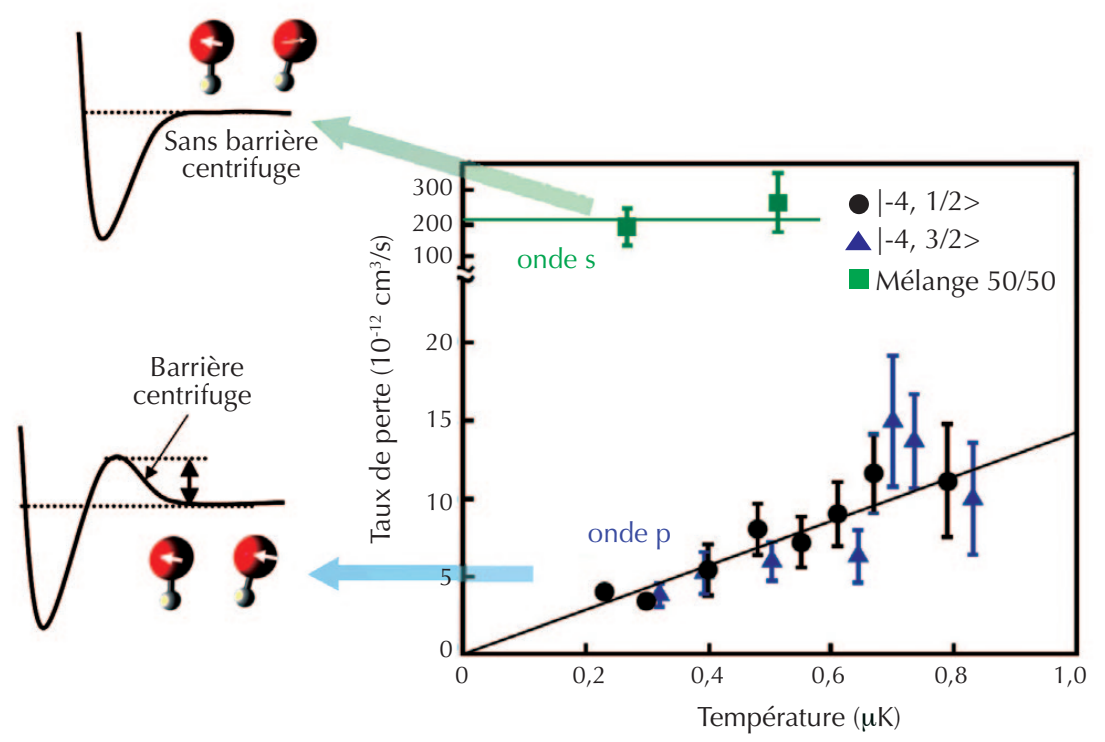

4. Mesure du taux de perte des molécules ${ }^{40} \mathrm{~K}^{87} \mathrm{Rb}$ dans la réaction $2 \mathrm{KRb} \rightarrow \mathrm{K}_{2}+\mathrm{Rb}_{2}$, en fonction de la température du gaz ultrafroid, illustrant le rôle de la statistique quantique en chimie ultrafroide. Si les molécules fermioniques sont dans le même état hyperfin (noté $|-4,1 / 2>0 u|-4,3 / 2>$ ), elles entrent en collision dans l'onde « $p$ » et doivent passer par effet tunnel à travers une barrière centrifuge pour réagir. Le taux observé est donc faible et proportionnel à la température. Si les molécules sont dans un mélange (noté 50/50) de ces états hyperfins, la réaction se produit sans barrière, en onde " s », et le taux est élevé et constant. Les lignes continues illustrent le bon accord entre théorie et expérience. (Adapté de Ospelkaus et al., Science 327 (2010) 853.)

L'excellente résolution en énergie du processus de transfert cohérent à deux photons (fig. 2), combinée à l'utilisation de rayonnement micro-onde, autorise la création de molécules KRb dans un état sélectionné parmi les 36 états hyperfins possibles. Lorsque deux molécules KRb dans leur état hyperfin fondamental (d'énergie la plus basse) entrent en collision, on montre que seule la réaction avec échange d'atomes est énergétiquement possible $: \mathrm{KRb}+\mathrm{KRb} \rightarrow \mathrm{K}_{2}+\mathrm{Rb}_{2}$. Cette réaction chimique ultrafroide est détectée par la perte de KRb dans le piège. Le taux mesuré est suffisamment important pour conclure qu'il n'y a pas de barrière de réaction, contrairement à de nombreuses réactions habituellement observées en chimie.

\section{Le rôle de la statistique quantique}

Décrire cette réaction chimique dans tous ses détails est une tâche ardue. Un modèle particulièrement simple, mais assez précis, consiste à séparer l'espace en deux parties. Si deux molécules se rapprochent à des distances suffisamment petites pour que l'interaction chimique très complexe soit dominante, on admet alors qu'elles réagissent avec une probabilité égale à 1 . Seule la partie à longue portée de la collision, où les interactions restent très faibles, est alors calculable exactement dans le cadre d'un modèle quantique : les molécules ultrafroides se rapprochent avec un moment orbital relatif quantifié en ondes partielles $l=0,1,2 \ldots$, engendrant une barrière de potentiel dite "centrifuge " qui empêche alors les molécules de s'approcher, sauf lorsque la barrière est très basse $(l=1)$ ou inexistante $(l=0)$.

$\mathrm{La}$ combinaison isotopique choisie fait de ${ }^{40} \mathrm{~K}^{87} \mathrm{Rb}$ une molécule fermionique. La fonction d'onde totale d'une paire de telles molécules créées dans le même état interne (comme l'état hyperfin fondamental) doit être antisymétrique par rapport à l'échange des molécules, ce qui impose que la partie de la fonction d'onde décrivant leur mouvement relatif soit ellemême antisymétrique, correspondant à l'onde partielle $l=1$ (ou onde « $p »$ ). Cependant, l'énergie de collision est si faible en régime ultrafroid que les molécules doivent traverser cette barrière centrifuge par effet tunnel. L'expérience montre que le taux de réaction croît linéairement avec la température (fig. 4), conformément aux lois de seuil évoquées plus haut. Si, au contraire, les molécules sont créées dans des états hyperfins différents, cette condition d'antisymétrie de la fonction d'onde totale n'est plus requise : les collisions ont lieu majoritairement dans l'onde "s » $(l=0)$ sans barrière centrifuge, conduisant à un taux de réaction plus important et indépendant de la température (fig. 4), comme prévu par les lois de seuil.

\section{L’anisotropie des interactions dipolaires}

L'interaction dipôle-dipôle dans les gaz de molécules polaires ultrafroides peut être activée par un champ électrique externe, qui tend à orienter le moment dipolaire électrique des molécules dans le référentiel du laboratoire. Un champ électrique modeste de quelques $\mathrm{kV} / \mathrm{cm}$ appliqué à un gaz de $\mathrm{KRb}$, induit ainsi un moment dipolaire $\mathrm{d}=0,22$ Debye (à comparer à la valeur maximale de 0,5 Debye dans le référentiel propre). Ces expériences n'ont pas encore atteint le régime de gaz quantique dégénéré. Les collisions entre molécules sont dominées par un effet principal : l'attraction entre moments dipolaires induits orientés en configuration tête-à-queue $(\rightarrow \rightarrow)$, qui compense la barrière centrifuge. Au-dessus de $\mathrm{d}=0,1$ Debye, le taux de réaction crô̂t en $\mathrm{d}^{6}$ et, à $\mathrm{d}=0,22$ Debye, il est presque 100 fois plus grand qu'à $\mathrm{d}=0,1$ Debye (triangles bleus sur la figure $5 \mathrm{a}$ ).

Ce résultat représente une remarquable démonstration de contrôle du taux d'une réaction chimique en régime ultrafroid. Mais il constitue aussi un sérieux obstacle à la création d'un gaz quantique dégénéré, puisque les molécules sont progressivement détruites. La solution découverte et mise en œuvre au JILA pour éliminer les collisions en tête-à-queue, consiste à confiner en 
>>

réseau optique ${ }^{\star}$ les molécules polaires dans des plans perpendiculaires au champ électrique d'orientation (fig. 5c). L'effet combiné de la répulsion entre moments dipolaires orientés en configuration côte-à-côte $(\uparrow \uparrow)$ et de la statistique fermionique qui empêche les molécules de se rapprocher, conduit à un taux de réaction qui n'augmente plus lorsque le champ électrique (et donc le moment dipolaire induit) augmente (points noirs sur la figure 5a).

\section{Perspectives}

Les progrès considérables accomplis dans la production de molécules ultrafroides ont fait émerger ce domaine de recherches à la fois fondamentales et interdisciplinaires. Ces travaux s'inscrivent dans le développement continu de méthodes de contrôle ultime de systèmes quantiques de plus en plus complexes.

La possibilité de contrôler une réaction chimique ultrafroide impliquant des effets purement quantiques est maintenant clairement démontrée. La prochaine étape majeure consistera à détecter les produits de la réaction, qui permettront de fournir une représentation complète du processus. En revanche, la possibilité de supprimer ces réactions au moyen d'un champ électrique externe, combinée à la compréhension détaillée des collisions élastiques et inélastiques, ouvrent la voie à l'étude d'une nouvelle matière quantique constituée de molécules polaires. Par exemple, l'empilement en couches de structures planaires telles que celles représentées en figure $5 c$, mènera à l'observation de nouveaux effets de forte corrélation entre particules, résultant de la compétition entre répulsion moléculaire à l'intérieur d'une couche, et attraction entre molécules localisées dans deux couches voisines.

L'ordre spatial engendré dans le gaz quantique par le piégeage des molécules dans un réseau optique tridimensionnel permettra de simuler les hamiltoniens de Bose-Hubbard ou de Fermi-Hubbard de la matière condensée, qui décrivent l'interaction entre spins électroniques localisés aux sites d'une matrice cristalline.

Enfin, même en l'absence de confinement externe, les molécules polaires pourraient s'auto-assembler pour former une structure cristalline dès lors que leurs interactions à longue portée sont suffisamment fortes et répulsives.

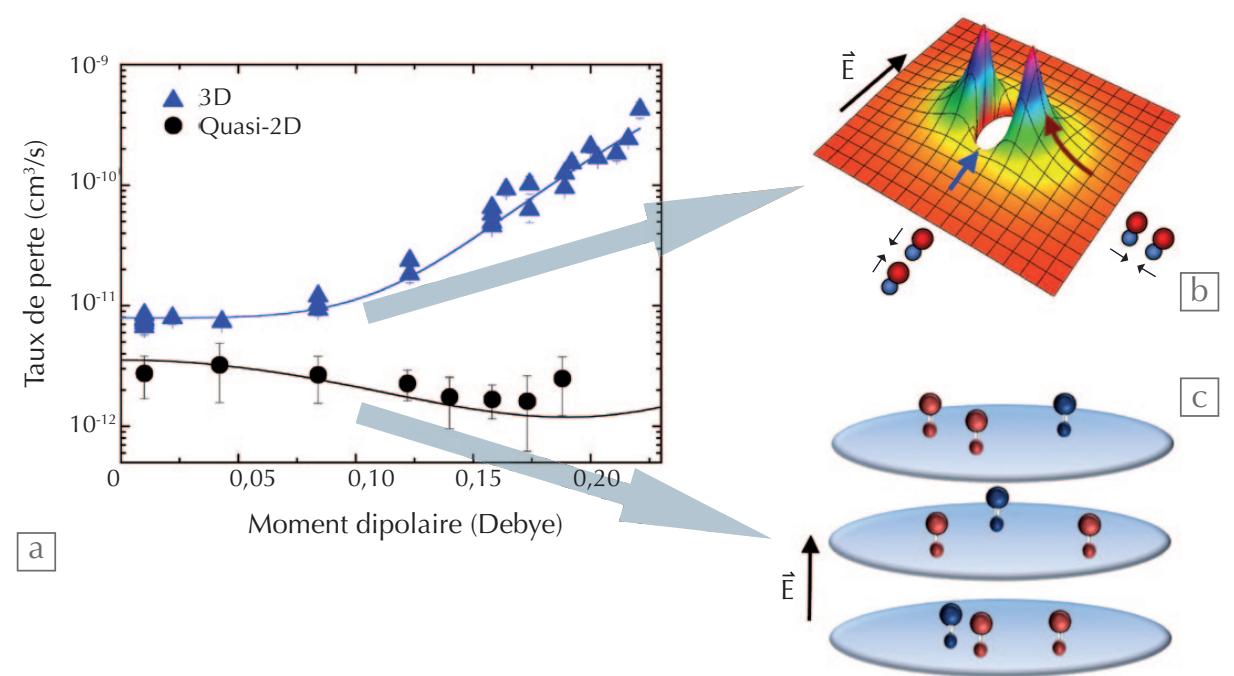

5. Mesure du taux de perte de ${ }^{40} K^{87} R b$ dans la réaction $2 K R b \rightarrow K_{2}+R b_{2}$, en fonction du moment dipolaire induit par le champ électrique externe E. Sans confinement spatial (noté « 3D »), les molécules entrent en collision et réagissent de préférence en configuration « tête-à-queue » sans rencontrer de barrière de potentiel. Le taux augmente alors avec le moment dipolaire induit. Avec confinement (noté " quasi-2D »), les molécules sont confinées dans des plans superposés et ne peuvent interagir qu’en configuration « côte-à-côte » qui induit une interaction répulsive, et donc une diminution du taux de réaction. (Adapté de Ni et al., Nature 464 (2010) 1324, et de Miranda et al., Nature Phys. 7 (2011) 502.)

\section{Glossaire}

Molécule polaire : qui possède un moment électrique dipolaire intrinsèque comme, par exemple, la molécule diatomique hétéronucléaire KRb.

Réseau optique : désigne la structure périodique spatiale de l'énergie potentielle d'une ou plusieurs ondes électromagnétiques stationnaires, qui permet le piégeage de particules ultrafroides neutres à des emplacements régulièrement espacés, comme ils le seraient dans un solide.

Gaz ultrafroid dense : notons que ces densités restent très faibles par comparaison à la densité atmosphérique.

L'espace des phases désigne l'espace à $6 \mathrm{~N}$ dimensions engendré par les $3 \mathrm{~N}$ coordonnées spatiales et les $3 \mathrm{~N}$ composantes de l'impulsion d'un ensemble de $\mathrm{N}$ particules. En mécanique quantique, une cellule de l'espace des phases a une taille déterminée par le principe d'incertitude de Heisenberg. Une densité de 1 dans cet espace est la condition requise pour observer la dégénérescence d'un gaz quantique.

Niveau ro-vibrationnel : désigne un niveau d'énergie moléculaire de vibration et de rotation.

Résonance de Fano-Feshbach : type de résonance collisionnelle qui se produit lorsque l'énergie d'un état moléculaire faiblement lié est égale à l'énergie cinétique d'une paire d'atomes en collision. En régime ultrafroid, ces résonances présentent l'immense intérêt d'être ajustables à l'aide d'un champ magnétique modéré, permettant ainsi le contrôle des propriétés collisionnelles des particules.

Pour en savoir plus, le lecteur intéressé pourra consulter le site internet de l'équipe du JILA : http://jila.colorado. edu/yelabs/research/ultracold-molecules .

Traduit de l'anglais et adapté pour Reflets de la physique par Amodsen Chotia, qui a été postdoctorant sur l'expérience $\mathrm{KRb}$ dans les groupes de Jun Ye et Deborah Jin, et Olivier Dulieu, membre du comité de rédaction de Reflets de la Physique.

\section{Remerciements}

D. S. Jin et J. Ye remercient leurs collègues du JILA (Boulder, CO, USA) et leurs collaborateurs extérieurs qui ont rendu ce travail possible, ainsi que le NIST, la NSF, l'AFOSR et le ministère de l'Énergie (USA) pour leur soutien financier.

\section{Références}

1• J. Doyle et al., eds., special issue, "Ultracold Polar Molecules: Formation and Collisions," Eur. Phys. J. D 31 (2004) ; L. D. Carr, J. Ye, eds., New J. Phys. 11 (2009) 055009 ; R. V. Krems, W. C. Stwalley, B. Friedrich, eds., Cold Molecules: Theory, Experiment, Applications, CRC Press, Boca Raton, FL (2009).

2• L. D. Carr et al., New J. Phys. 11 (2009) 055049.

3• 0. Dulieu et C. Gabbanini, Rep. Prog. Phys. 72 (2009) 086401.

4• M.A. Baranov, Phys. Reports 464 (2008) 71.

5• T. Lahaye et al., Rep. Prog. Phys. 72 (2009) 126401.

6• R.V. Krems, Phys. Chem. Chem. Phys. 10 (2008) 4079.

7• J.L. Miller, Physics Today 66 (2013) 12. 